\title{
INCLUSION AND INTERSECTION THEOREMS WITH APPLICATIONS IN EQUILIBRIUM THEORY IN $G$-CONVEX SPACES
}

\author{
Mircea Balaj and Donal O'Regan
}

\begin{abstract}
In this paper we obtain a very general theorem of $\rho$-compatibility for three multivalued mappings, one of them from the class $\mathfrak{B}$. More exactly, we show that given a $G$-convex space $Y$, two topological spaces $X$ and $Z$, a (binary) relation $\rho$ on $2^{Z}$ and three mappings $P: X \multimap Z$, $Q: Y \multimap Z$ and $T \in \mathfrak{B}(Y, X)$ satisfying a set of conditions we can find $(\widetilde{x}, \widetilde{y}) \in X \times Y$ such that $\widetilde{x} \in T(\widetilde{y})$ and $P(\widetilde{x}) \rho Q(\widetilde{y})$. Two particular cases of this general result will be then used to establish existence theorems for the solutions of some general equilibrium problems.
\end{abstract}

\section{Introduction}

Since Park and Kim introduced the concept of generalized convex space (simply, $G$-convex space) [30], a large number of works contributing mainly to the KKM theory and equilibrium theory on these spaces have appeared. On the other hand, in the fixed point theory of multivalued mappings, Park introduced a new class of multivalued mappings, called the "better" admissible class $\mathcal{B}$, first in topological vector spaces [23] and later in $G$-convex spaces [25].

In this paper, after a preliminary section, we obtain a very general theorem of $\rho$-compatibility for three multivalued mappings, one of them from the class $\mathfrak{B}$. More exactly, we show that given a $G$-convex space $Y$, two topological spaces $X$ and $Z$, a (binary) relation $\rho$ on $2^{Z}$ and three mappings $P: X \multimap Z, Q: Y \multimap Z$ and $T \in \mathfrak{B}(Y, X)$ satisfying a set of conditions we can find $(\widetilde{x}, \widetilde{y}) \in X \times Y$ such that $\widetilde{x} \in T(\widetilde{y})$ and $P(\widetilde{x}) \rho Q(\widetilde{y})$. Two particular cases of this general result will be then used to establish existence theorems for the solutions of some general equilibrium problems described below.

In many recent papers (see [1], [2], [4], [8]-[10], [12], [13], [15], [17]-[21], [29]) and the references therein) one studies one of more of the following equilibrium problems:

Received January 12, 2009; Revised June 4, 2009.

2000 Mathematics Subject Classification. 54C60, 54H25, 91B50.

Key words and phrases. $G$-convex space, the better admissible class, fixed point, equilibrium problems. 
For a suitable choice of the sets $X, Z$ and $V$ and of the maps $F: X \times Z \multimap V$ and $C: X \multimap V$ we are interesting in finding $x_{0} \in X$ such that one of the following situations occurs:

(I) $F\left(x_{0}, z\right) \subseteq C\left(x_{0}\right)$ for all $z \in Z$;

(II) $F\left(x_{0}, z\right) \cap C\left(x_{0}\right) \neq \emptyset$ for all $z \in Z$;

(III) $F\left(x_{0}, z\right) \nsubseteq C\left(x_{0}\right)$ for all $z \in Z$;

(IV) $F\left(x_{0}, z\right) \cap C\left(x_{0}\right)=\emptyset$ for all $z \in Z$.

Each existence result concerning problem (I) (respectively, (II)) yields an existence theorem for problem IV (respectively III), if we take into account the following equivalences: $F(x, z) \subseteq C(x) \Leftrightarrow F(x, y) \cap(V \backslash C(x))=\emptyset$ and $F(x, z) \cap C(x) \neq \emptyset \Leftrightarrow F(x, z) \nsubseteq(V \backslash C(x))$. For this reason we can fix our attention on problems (I) and (II), only.

In [3] and [4] the first author extends and unifies these problems by considering a (binary) relation $\rho$ on $2^{Z}$ and looking for a point $x_{0} \in X$ such that $F\left(x_{0}, z\right) \rho C\left(x_{0}\right)$ for all $z \in Z$. In Section 4 the following more general problem is considered:

Let $(Y, D ; \Gamma)$ and $\left(Z, D^{\prime} ; \Gamma^{\prime}\right)$ be $G$-convex spaces, $X$ and $V$ be topological spaces, $C: X \multimap V, F: X \times Z \multimap V$ and $Q: Y \multimap Z$ be three mappings and $\rho$ be a relation on $2^{V}$. Find $x_{0} \in X$ such that

(V) $F\left(x_{0}, z\right) \rho C\left(x_{0}\right)$ for all $z \in Q(D)$.

In a very recent paper [5] the authors study the following problem:

Given a $G$-convex space $(Y, D ; \Gamma)$, two topological spaces $Z$ and $V$, three mappings $P: Y \multimap Z, F: Y \times Z \multimap V, C: Z \multimap V$ and a relation $\rho$ on $2^{V}$, find $y_{0} \in Y$ such that

$$
\forall u \in D \exists z \in P\left(y_{0}\right) \text { such that } F(u, z) \rho C(z),
$$

and, respectively

$$
\forall y \in Y \quad \exists z \in P\left(y_{0}\right) \text { such that } F(y, z) \rho C(z) .
$$

Each of these two problems is called, in the paper mentioned above, weak $\rho$ equilibrium problem with respect to $P$. In the last section of the paper the two problems will be generalized as follows:

Given a $G$-convex space $(Y, D ; \Gamma)$, three topological spaces $X, Z$ and $V$, three mappings $P: X \multimap Z, F: Y \times Z \multimap V, C: Z \multimap V$ and a relation $\rho$ on $2^{V}$, find $x_{0} \in X$ such that

$\left(V I_{1}\right) \forall u \in D \quad \exists z \in P\left(x_{0}\right)$ such that $F(u, z) \rho C(z)$, and, respectively

$\left(V I_{2}\right) \forall y \in Y \quad \exists z \in P\left(x_{0}\right)$ such that $F(y, z) \rho C(z)$.

\section{Preliminaries}

Let $X$ and $Y$ be nonempty sets. A multivalued mapping (or simply, a mapping) $T: X \multimap Y$ is a function from $X$ into the power set of a set $Y$. If $y \in Y$, the sets $T^{-}(y)=\{x \in X: y \in T(x)\}, T^{*}(y)=\{x \in X: y \notin T(x)\}$ are called the fiber and respectively, the cofiber of $T$ on $y$. For $A \subseteq X, T(A)=$ 
$\bigcup_{x \in A} T(x)$ is the image of $A$ under $T$. If $X$ and $Y$ are topological spaces a mapping $T: X \multimap Y$ is said to be: (i) upper semicontinuous (in short, u.s.c) (respectively, lower semicontinuous (in short, l.s.c.)) if for every closed subset $B$ of $Y$ the set $\{x \in X: T(x) \cap B \neq \emptyset\}$ (respectively, $\{x \in X: T(x) \subseteq B\}$ ) is closed; (ii) closed if its graph (that is, the set $\operatorname{Gr} T=\{(x, y) \in X \times Y: y \in$ $T(x)\}$ ) is a closed subset of $X \times Y$; (iii) compact if $T(X)$ is contained in a compact subset of $Y$.

The following lemma collects known facts about u.s.c. or l.s.c. mappings (see for instance [16] for assertions (i) (ii) and (iii), respectively [31] for assertion (iv)).

Lemma 1. Let $X$ and $Y$ be topological spaces and $T: X \multimap Y$ be a mapping.

(i) If $Y$ is compact and $T$ is closed, then $T$ is u.s.c..

(ii) If $Y$ is regular and $T$ is u.s.c. with closed values, then $T$ is closed.

(iii) If $X$ is compact and $T$ is u.s.c. with compact values, then $T(X)$ is compact.

(iv) $T$ is l.s.c. if and only if for any $x \in X, y \in T(x)$ and any net $\left\{x_{\alpha}\right\}$ converging to $x$, there exists a net $\left\{y_{\alpha}\right\}$ converging to $y$, with $y_{\alpha} \in$ $T\left(x_{\alpha}\right)$ for each $\alpha$.

A generalized convex space or a $G$-convex space $(Y, D ; \Gamma)$ (see $[28])$ consists of a topological space $Y$ and a nonempty set $D$ such that for each $A \in\langle D\rangle$ with the cardinality $|A|=n+1$ there exists a subset $\Gamma(A)$ of $Y$ and a continuous function $\Phi_{A}: \Delta_{n} \rightarrow \Gamma(A)$ such that $J \in\langle A\rangle$ implies $\Phi_{A}\left(\Delta_{J}\right) \subseteq \Gamma(J)$. Here $\langle D\rangle$ denotes the set of all nonempty finite subsets of $D, \Delta_{n}$ denotes the standard $n$-dimensional simplex with vertices $\left\{e_{i}\right\}_{i=0}^{n}$ and $\Delta_{J}$ the face of $\Delta_{n}$ corresponding to $J$; that is, if $A=\left\{u_{0}, u_{1}, \ldots, u_{n}\right\}$ and $J=\left\{u_{i_{0}}, u_{i_{1}}, \ldots, u_{i_{k}}\right\}$ $\subseteq A$, then $\Delta_{J}=\operatorname{co}\left\{e_{i_{0}}, e_{i_{1}}, \ldots, e_{i_{k}}\right\}$. When $D$ is a subset of $Y$, as matters stands throughout this paper, a subset $C$ of $Y$ is said to be $G$-convex if for each $A \in\langle C \cap D\rangle, \Gamma(A) \subseteq C$.

The main example of $\bar{G}$-convex space corresponds to the case when $Y=D$ is a convex subset of a topological vector space, and for each $A \in\langle A\rangle, \Gamma(A)$ is the convex hull of $A$. For other examples of $G$-convex see [26] and [27].

Let $X$ be a topological space and $(Y, D ; \Gamma)$ be a $G$-convex space. The better admissible class $\mathfrak{B}$ of mappings from $Y$ into $X$ (see [25]) is defined as follows:

$T \in \mathfrak{B}(Y, X) \Leftrightarrow T: Y \multimap X$ is a mapping such that for any $A \in\langle D\rangle$ with the cardinality $|A|=n+1$ and any continuous mapping $p: T(\Gamma(A)) \rightarrow \Delta_{n}$ the composition $p \circ T_{\mid \Gamma(A)} \circ \phi_{A}: \Delta_{n} \multimap \Delta_{n}$ has a fixed point. The class $\mathfrak{B}(Y, X)$ includes many important classes of mappings, such as $\mathfrak{A}_{c}^{\kappa}(Y, X)$ in [24], $K K M(Y, X)$ in [7] and $A(Y, X)$ in [6], as proper subclasses.

As usual, for a subset $A$ of a topological space, we denote the interior and the closure of $A$ by int $A$ and $\bar{A}$, respectively. From now on all topological spaces will be assumed Hausdorff. 


\section{Inclusion and intersection theorems}

In this section, unless otherwise specified, we shall assume that $(Y, D ; \Gamma)$ is a $G$-convex space, $X$ and $Z$ are topological spaces, $T \in \mathfrak{B}(Y, X)$ is compact, $P: X \multimap Z, Q: Y \multimap Z$ are two mappings and $\rho$ is a (binary) relation on $2^{Z}$. Motivated by the considerations from the previous section we will consider the following two particular relations on $2^{Z}$ : if $A, B \subseteq Z$, then

(i) $A \rho_{1} B \Leftrightarrow A \subseteq B$;

(ii) $A \rho_{2} B \Leftrightarrow A \cap B \neq \emptyset$;

Denote by $\rho^{c}$ the complementary relation of $\rho$, that is, for any $A, B \subseteq Z$ exactly one of the following assertions $A \rho B, A \rho^{c} B$ holds.

Theorem 2. Suppose that the following conditions are satisfied:

(i) $X=\bigcup_{u \in D}$ int $\{x \in X: P(x) \rho Q(u)\}$;

(ii) for each $x \in X,\{y \in Y: P(x) \rho Q(y)\}$ is G-convex.

Then there exists $(\widetilde{x}, \widetilde{y}) \in X \times Y$ such that $\widetilde{x} \in T(\widetilde{y})$ and $P(\widetilde{x}) \rho Q(\widetilde{y})$.

Proof. For $u \in D$ set $S(u)=\{x \in X: P(x) \rho Q(u)\}$. Let $X_{0}=\overline{T(Y)}$. Since $X_{0}$ is compact there exists a finite set $A=\left\{u_{0}, u_{1}, \ldots, u_{n}\right\} \subseteq D$ such that $X_{0}=\bigcup_{i=0}^{n}\left(\right.$ int $\left.S\left(u_{i}\right) \cap X_{0}\right)$. Let $\left\{\alpha_{0}, \alpha_{1}, \ldots, \alpha_{n}\right\}$ be a partition of unity on $X_{0}$ subordinated to the cover $\left\{\operatorname{int} S\left(u_{i}\right) \cap X_{0}: 0 \leq i \leq n\right\}$. Recall that this means that

$$
\left\{\begin{array}{l}
\alpha_{i}: X_{0} \rightarrow[0,1] \text { is continuous for each } i \in\{0,1, \ldots, n\} \\
\alpha_{i}(x)>0 \Rightarrow x \in \operatorname{int} S\left(u_{i}\right) \\
\sum_{i=0}^{n} \alpha_{i}(x)=1 \text { for each } x \in X_{0}
\end{array}\right.
$$

Define $f: T(\Gamma(A)) \rightarrow \Delta_{n}$ by

$$
f(x)=\sum_{i=0}^{n} \alpha_{i}(x) e_{i} \text { for all } x \in T(\Gamma(A)) .
$$

Clearly $f$ is continuous. Since $(Y, D ; \Gamma)$ is a $G$-convex space there exists a continuous function $\phi_{A}: \Delta_{n} \rightarrow \Gamma(A)$ such that $\phi_{A}\left(\Delta_{J}\right) \subset \Gamma(J)$ for each $J \in$ $\langle A\rangle$. Since $T \in \mathfrak{B}(Y, X)$, there exists $\widetilde{t} \in \Delta_{n}$ such that $\widetilde{t} \in\left(f \circ T_{\mid \Gamma(A)} \circ \phi_{A}\right)(\widetilde{t})$. Let $\widetilde{y}=\phi_{A}(\widetilde{t})$. Then $\widetilde{y} \in\left(\phi_{A} \circ f \circ T\right)(\widetilde{y})$. Thus for some $\widetilde{x} \in T(\widetilde{y})$ we have $\widetilde{y}=\phi_{A}(f(\widetilde{x}))$. Let $\widetilde{J}=\left\{i \in\{0,1, \ldots, n\}: \alpha_{i}(\widetilde{x})>0\right\}$. Then $f(\widetilde{x}) \in \Delta_{\widetilde{J}}$ and

$$
\widetilde{y}=\phi_{A}(f(\widetilde{x})) \in \phi_{A}\left(\Delta_{\widetilde{J}}\right) \subset \Gamma\left(\left\{u_{i}: i \in \widetilde{J}\right\}\right) .
$$

For each $i \in \widetilde{J}$ we have $\widetilde{x} \in \operatorname{int} S\left(u_{i}\right) \subset S\left(u_{i}\right)$, hence $\left\{u_{i}: i \in \widetilde{J}\right\} \subset S^{-}(\widetilde{x})$. By (ii) and (1) it follows that $\widetilde{y} \in S^{-}(\widetilde{x})$, that is $P(\widetilde{x}) \rho Q(\widetilde{y})$ and the proof is complete.

Remark 1. In the particular case $X=Z, P=1_{X}$ and $\rho$ is either $\rho_{1}$ or $\rho_{2}$ the previous theorem generalizes Theorems 2.5 and 2.6 in [17], 3.1 and 3.5 in [18], and 3.1 in [20]. In the above mentioned results $Y$ is a convex set in a topological vector space and $T \in K K M(Y, X)$. Also a particular case of Theorem 2 can 
be compared with some closely related results obtained in $F C$-spaces by Ding (Theorems 4.4 in [8] and 3.1 in [9]) and Fang and Huang (Theorem 3.1 in [14]).

As usual the compactness hypothesis on $T$ can be replaced by some coercivity conditions. The next result is an example in this direction.

Theorem 3. Suppose that condition (i) and (ii) in Theorem 2 hold. Moreover, assume that $\overline{T(Y)}$ is paracompact and there exist a compact subset $K$ of $X$ and $B \in\langle D\rangle$ such that

$$
X \backslash K \subset \bigcup_{u \in B} \operatorname{int}\{x \in X: P(x) \rho Q(u)\} .
$$

Then there exists $(\widetilde{x}, \widetilde{y}) \in X \times Y$ such that $\widetilde{x} \in T(\widetilde{y})$ and $P(\widetilde{x}) \rho Q(\widetilde{y})$.

Proof. Since $K$ is compact there exists $B^{\prime} \in\langle D\rangle$ such that $K \subset \bigcup_{u \in B^{\prime}}$ int $\{x \in$ $X: P(x) \rho Q(u)\}$. Let $A=B \cup B^{\prime}$. We have $Y=\bigcup_{u \in A}$ int $\{x \in X$ : $P(x) \rho Q(u)\}$. Keeping the notations from the previous proof, $\mathcal{U}=\{\operatorname{int} S(u) \cap$ $\left.X_{0}: u \in A\right\}$ is an open cover of $X_{0}$. Since $X_{0}$ is paracompact, there exists a partition of unity on $X_{0}$ subordinates to $\mathcal{U}$ and the proof is now similar to that of Theorem 2.

We say that a mapping $P: X \multimap Z$ is $\rho$-semicontinuous if for each open subset $G$ of $Z$ the set $\{x \in X: P(x) \rho G\}$ is open.

Remark 2. It's clear that $\rho_{1}$ (respectively, $\rho_{2}$ )-semicontinuity means upper (respectively, lower)-semicontinuity.

Theorem 4. Assume that the following conditions are fulfilled:

(i) for each $x \in X$ there exists $u \in D$ such that $P(x) \rho Q(u)$;

(ii) $P$ is $\rho$-semicontinuous;

(iii) $Q_{\mid D}$ has open values;

(iv) for each $x \in X,\{y \in Y: P(x) \rho Q(y)\}$ is G-convex.

Then there exists $(\widetilde{x}, \widetilde{y}) \in X \times Y$ such that $\widetilde{x} \in T(\widetilde{y})$ and $P(\widetilde{x}) \rho Q(\widetilde{y})$.

Proof. By (i), $X=\bigcup_{u \in D}\{x \in X: P(x) \rho Q(u)\}$. By (ii) and (iii), for each $u \in D,\{x \in X: P(x) \rho Q(u)\}$ is open. Thus, condition (i) in Theorem 2 is fulfilled. The conclusion follows from Theorem 2 .

When $\rho=\rho_{1}$, Theorem 4 yields the following result:

Corollary 5. Assume that the following conditions are fulfilled:

(i) for each $x \in X$ there exists $u \in D$ such that $P(x) \subseteq Q(u)$;

(ii) $P$ is u.s.c;

(iii) $Q$ has $G$-convex fibers and $Q_{\mid D}$ has open values.

Then there exists $(\widetilde{x}, \widetilde{y}) \in X \times Y$ such that $\widetilde{x} \in T(\widetilde{y})$ and $P(\widetilde{x}) \subseteq Q(\widetilde{y})$. 
Proof. The desired conclusion follows from Theorem 4 as soon as we prove that for $\rho=\rho_{1}$ the requirement (iv) in Theorem 4 is fulfilled. Let $x \in X$ and $A \in\langle\{u \in D: P(x) \subseteq Q(u)\}\rangle$. Suppose that for some $y \in \Gamma(A)$ there exists $z \in$ $P(x) \backslash Q(y)$. For each $u \in A, z \in P(x) \subseteq Q(u)$, hence $A \subset Q^{-}(z)$. Since $Q^{-}(z)$ is $G$-convex, it follows that $y \in Q^{-}(z)$, that is $z \in Q(y)$. The contradiction obtained show that the set $\{y \in Y: P(x) \subseteq Q(y)\}$ is $G$-convex.

Definition 1. A mapping $Q: Y \multimap Z$ is said to be:

(a) G-strongly quasiconvex if for each $A \in\langle D\rangle$ and any $y \in \Gamma(A)$, there is $u \in A$ such that $Q(u) \subseteq Q(y)$.

(b) $G$-quasiconvex [22] if $Z$ is a $G$-convex space and for each subset $G$-convex $M$ of $Z$ the set $\{y \in Y: Q(y) \cap M \neq \emptyset\}$ is $G$-convex.

It's clear that a mapping $G$-strongly quasiconvex is $G$-quasiconvex.

The next result is obtained from Theorem 4 , in a similar manner, when $Z$ is a $G$-convex space and $\rho=\rho_{2}$.

Corollary 6. Suppose that $\left(Z, \Gamma^{\prime} ; D^{\prime}\right)$ is a $G$-convex space and the following conditions are fulfilled:

(i) for each $x \in X$ there exists $u \in D$ such that $P(x) \cap Q(u) \neq \emptyset$;

(ii) $P$ is l.s.c.;

(iii) $Q_{\mid D}$ has open values;

(iv) either $Q$ is G-strongly quasiconvex or $Q$ is $G$-quasiconvex and $P$ is $G$-convex valued.

Then there exists $(\widetilde{x}, \widetilde{y}) \in X \times Y$ such that $\widetilde{x} \in T(\widetilde{y})$ and $P(\widetilde{x}) \cap Q(\widetilde{y}) \neq \emptyset$.

Remark 3. Corollary 6 generalizes Theorem 4 in [4].

\section{Existence results for Problem (V)}

Throughout this section, unless otherwise specified, we shall assume that $(Y, D ; \Gamma)$ and $\left(Z, D^{\prime} ; \Gamma^{\prime}\right)$ are $G$-convex spaces, $X$ and $V$ are topological spaces, $C: X \multimap V, F: X \times Z \multimap V$ and $Q: Y \multimap Z$ are three mappings and $\rho$ is a relation on $2^{V}$.

Theorem 7. Suppose that the following conditions are satisfied:

(i) for each $z \in Z$ the set $\{x \in X: F(x, z) \rho C(x)\}$ is closed;

(ii) $Q_{\mid D}$ has open values;

(iii) one of the following situations occurs:

(iii 1 ) $Q$ is strongly $G$-quasiconvex; or

(iii 2$) Q$ is $G$-quasiconvex and for each $x \in X$ the set $\{z \in Z$ : $\left.F(x, z) \rho^{c} C(x)\right\}$ is $G$-convex;

(iv) there exists a compact mapping $T \in \mathfrak{B}(Y, X)$ such that for each $y \in Y$, $x \in T(y)$ and $z \in Q(y)$ we have $F(x, z) \rho C(x)$.

Then there exists $x_{0} \in X$ such that $F\left(x_{0}, z\right) \rho C\left(x_{0}\right)$ for all $z \in Q(D)$. 
Proof. Let $P: X \multimap Z$ be the mapping defined by

$$
P(x)=\left\{z \in Z: F(x, z) \rho^{c} C(x)\right\} .
$$

Assume that the conclusion does not hold. Then for each $x \in X$ there exists $u \in D$ and $z \in Q(u)$ such that $F(x, z) \rho^{c} C(x)$, hence $z \in P(x) \cap Q(u)$. Since $P$ has open fibers (by (i)), it follows easily that $P$ is l.s.c.. By (iii), it follows that condition (iv) in Corollary 6 is fulfilled. By Corollary 6 , there exists $(\widetilde{x}, \widetilde{y}) \in$ $X \times Y$ such that $\widetilde{x} \in T(\widetilde{y})$ and $P(\widetilde{x}) \cap Q(\widetilde{y}) \neq \emptyset$. Let $z \in P(\widetilde{x}) \cap Q(\widetilde{y})$. Then $z \in Q(\widetilde{y})$ and $F(\widetilde{x}, z) \rho^{c} C(\widetilde{x})$, which contradicts (iv).

The concepts introduced below extend to $G$-convex spaces the corresponding notions from vector spaces (see [18]).

Definition 2. Suppose that $V$ is a topological vector space. The mapping $F(x, \cdot)$ is said to be:

(i) $C(x)$-G-quasiconvex if for each $x \in X, A \in\left\langle D^{\prime}\right\rangle$ and $z \in \Gamma^{\prime}(A)$ there exists $u^{\prime} \in A$ such that $F\left(x, u^{\prime}\right) \subseteq F(x, z)+C(x)$;

(ii) $C(x)$-G-quasiconvex-like if for each $x \in X, A \in\left\langle D^{\prime}\right\rangle$ and $z \in \Gamma^{\prime}(A)$ there exists $u^{\prime} \in A$ such that $F(x, z) \subseteq F\left(x, u^{\prime}\right)-C(x)$.

Theorem 8. Assume that:

(i) $C$ is a closed mapping;

(ii) for each $z \in Z, F(\cdot, z)$ is l.s.c.;

(iii) $Q_{\mid D}$ has open values;

(iv) one of the following situations holds:

(iv 1$) Q$ is $G$-strongly quasiconvex;

$\left(\mathrm{iv}_{2}\right) Q$ is $G$-quasiconvex and for each $x \in X F(x, \cdot)$ is $G$-strongly quasiconvex;

(iv 3$) V$ is a G-convex space, $Q$ is $G$-quasiconvex, and for each $x \in X$, the set $V \backslash C(x)$ is $G$-convex and the mapping $F(x, \cdot)$ is $G$ quasiconvex;

$\left(\mathrm{iv}_{4}\right) V$ is a topological vector space, $Q$ is $G$-quasiconvex, for each $x \in X C(x)$ is a convex cone and $F(x, \cdot)$ is $C(x)$-G-quasiconvex;

(v) there exists a compact mapping $T \in \mathfrak{B}(Y, X)$ such that for each $y \in Y$, $x \in T(y)$ and $z \in Q(y)$ we have $F(x, z) \subseteq C(x)$.

Then there exists $x_{0} \in X$ such that $F\left(x_{0}, z\right) \subseteq C\left(x_{0}\right)$ for all $z \in Q(D)$.

Proof. It suffices to prove that for $\rho=\rho_{1}$ conditions (i) and (iii) in Theorem 7 are fulfilled. Let $z \in Z$ and $x \in \overline{P^{*}(z)}$, where $P^{*}(z)=\{x \in X: F(x, z) \subseteq$ $C(x)\}$. Then there exists a net $\left\{x_{\alpha}\right\}_{\alpha \in \Lambda}$ in $P^{*}(z)$ such that $x_{\alpha} \rightarrow x$. For each $\alpha \in \Lambda, F\left(x_{\alpha}, z\right) \subseteq C\left(x_{\alpha}\right)$. Let $v \in F(x, z)$. By (ii), via Lemma 1 (iv), there is a net $\left\{v_{\alpha}\right\}_{\alpha \in \Lambda}$ in $V$ converging to $v$ such that $v_{\alpha} \in F\left(x_{\alpha}, z\right)$ for all $\alpha \in \Lambda$. Since $C: X \multimap V$ is closed, $v \in C(x)$ hence $F(x, z) \subseteq C(x)$. This shows that $\{x \in X: F(x, z) \subseteq C(x)\}$ is closed.

One can easily check that in each of the cases $\left(\mathrm{iv}_{2}\right)$ and $\left(\mathrm{iv}_{3}\right)$ for $x \in X$ arbitrarily fixed the set $\{z \in Z: F(x, z) \nsubseteq C(x)\}$ is $G$-convex and consequently 
in each of these cases condition (iii) in Theorem 7 holds. We show that the situation is the same in case $\left(\mathrm{iv}_{4}\right)$. Let $A \in\left\langle D^{\prime}\right\rangle$ such that for each $u^{\prime} \in A$, $F\left(x, u^{\prime}\right) \nsubseteq C(x)$. Suppose that for a $z \in \Gamma^{\prime}(A), F(x, z) \subseteq C(x)$. Since $F(x, \cdot)$ is $C(x)$-G-quasiconvex, for some $u^{\prime} \in A$ we have $F\left(x, u^{\prime}\right) \subseteq F(x, z)+C(x) \subseteq$ $C(x)+C(x)=C(x)$, which implies a contradiction.

Theorem 9. Suppose that $X$ is compact and the following conditions are satisfied:

(i) $C$ is a closed mapping;

(ii) for each $z \in Z, F(\cdot, z)$ is u.s.c. with nonempty compact values;

(iii) $Q_{\mid D}$ has open values;

(iv) one of the following situations holds:

(iv 1 ) $Q$ is $G$-strongly quasiconvex;

$\left(\mathrm{iv}_{2}\right) Q$ is $G$-quasiconvex and for each $x \in X F(x, \cdot)$ has $G$-convex cofibers;

$\left(\mathrm{iv}_{3}\right) V$ is a topological vector space, $Q$ is $G$-quasiconvex, for each $x \in X, C(x)$ is a convex cone and $F(x, \cdot)$ is $C(x)-G$-quasiconvex-like;

(v) there exists a compact mapping $T \in \mathfrak{B}(Y, X)$ such that for each $y \in Y$, $x \in T(y)$ and $z \in Q(y)$ we have $F(x, z) \cap C(x) \neq \emptyset$.

Then there exists $x_{0} \in X$ such that $F\left(x_{0}, z\right) \cap C\left(x_{0}\right) \neq \emptyset$ for all $z \in Q(D)$.

Proof. We show first that for $\rho=\rho_{2}$ condition (i) in Theorem 7 holds. Let $z \in Z$ and $x \in \overline{P^{*}(z)}$, where $P^{*}(z)=\{x \in X: F(x, z) \cap C(x) \neq \emptyset\}$. Then there exists a net $\left\{x_{\alpha}\right\}_{\alpha \in \Lambda}$ in $P^{*}(z)$ such that $x_{\alpha} \rightarrow x$. For each $\alpha \in \Lambda$ there is $v_{\alpha} \in F\left(x_{\alpha}, z\right) \cap C\left(x_{\alpha}\right)$. Since $F(\cdot, z)$ is u.s.c. with nonempty compact values and $X$ is compact, $F(X, z)=\bigcup_{x \in X} F(x, z)$ is compact hence without loss of generality we may suppose that $\left\{v_{\alpha}\right\}_{\alpha \in \Lambda}$ converges to $v \in F(X, z)$. The space $F(X, z)$ being compact it is regular and by Lemma 1 (ii), $F(\cdot, z)$ is closed. Hence $v \in F(x, z)$. Since $C$ is closed, it follows that $v \in C(x)$. Thus $v \in F(x, z) \cap C(x)$ hence $\{x \in X: F(x, z) \cap C(x) \neq \emptyset\}$ is closed.

We show that in each of the cases (iv ${ }_{2}$ ) and (iv 3 ) for $x \in X$ arbitrarily fixed, the set $\{z \in Z: F(x, z) \cap C(x)=\emptyset\}$ is $G$-convex and consequently in each of these cases condition (iii) in Theorem 7 holds.

Case $\left(\mathrm{iv}_{2}\right)$. Let $A \in\left\langle D^{\prime}\right\rangle$ such that for each $u^{\prime} \in A, F\left(x, u^{\prime}\right) \cap C(x)=\emptyset$. Suppose that for a $z \in \Gamma^{\prime}(A)$ there exists $v \in F(x, z) \cap C(x)$. Then $v \in C(x)$ and for each $u^{\prime} \in A$, since $F\left(x, u^{\prime}\right) \cap C(x)=\emptyset, v \notin F\left(x, u^{\prime}\right)$. It follows that $A \subseteq[F(x, \cdot)]^{*}(v)$. The cofiber $[F(x, \cdot)]^{*}(v)$ being convex, $z \in[F(x, \cdot)]^{*}(v)$. This implies $v \notin F(x, z)$; a contradiction.

Case (iv 3$)$. Let $A \in\left\langle D^{\prime}\right\rangle$ such that for each $u^{\prime} \in A, F\left(x, u^{\prime}\right) \cap C(x)=\emptyset$. Suppose that for a $z \in \Gamma^{\prime}(A), F(x, z) \cap C(x) \neq \emptyset$. Since $F(x, \cdot)$ is $C(x)-G$ quasiconvex-like, for some $u^{\prime} \in A$ we have $F(x, z) \subseteq F\left(x, u^{\prime}\right)-C(x)$, hence $\left(F\left(x, u^{\prime}\right)-C(x)\right) \cap C(x) \neq \emptyset$. This implies $\emptyset \neq F\left(x, u^{\prime}\right) \cap(C(x)+C(x))=$ $F\left(x, u^{\prime}\right) \cap C(x)$; a contradiction.

The desired conclusion follows now from Theorem 7 . 
Remark 4. When $Y$ and $Z$ are convex sets in topological vector spaces our theorems from this section can be compared with similar results obtained by Lin et al. such as Theorems 4.1-4.6 in [18] and 4.1-4.4 in [20]. However, there are at least two major differences between our results and the above mentioned results: 1) Lin's results involve only three mappings $F, C$ and $T ; 2)$ In the above mentioned results $T \in K K M(X, Y)$. This condition is stronger than $T \in \mathfrak{B}(X, Y)$. One can also compare Theorems 8 and 9 with Theorems 11 and 13 in [4] and Theorem 4.1 and Corollaries 4.1 and 4.3 in [14].

Example. The following example shows that although the assumptions of Theorems 8 and 9 are not always easy to verify in practice, these theorems could be important tools in establishing the existence of solutions for the corresponding problems.

Let $X=[-2,2], Y=D=[-1,1], Z=D^{\prime}=[-4,4], V=\mathbb{R}$ and for each $A \in\langle D\rangle$ (respectively, $\left.A \in\left\langle D^{\prime}\right\rangle\right) \Gamma(A)=\operatorname{co} A$ (respectively $\Gamma^{\prime}(A)=c o A$ ). Let the mappings $C: X \multimap V, F: X \times Z \multimap V, T: Y \multimap X$ and $Q: Y \multimap Z$ be defined by:

$$
\begin{gathered}
C(x)=\left[x^{2}-3, \infty\right), F(x, z)=\left[-z^{2}+2 x z+6, \infty\right), \\
T(y)=[y-1, y+1], Q(y)=(y-2, y+2) .
\end{gathered}
$$

Observe that $F(x, z) \subseteq C(x)$ if and only if $|x-z| \leq 3$. Since $T$ is a Kakutani mapping (that is, u.s.c with compact convex values) we have $T \in \mathfrak{B}(Y, X)$. For each $y \in Y, x \in T(y)$ and $z \in Q(y)$ we have $|x-z| \leq|x-y|+|y-z| \leq 3$, hence $F(x, z) \subseteq C(x)$. It is easy to check that all the requirements of Theorem 8 are fulfilled. An $x_{0}$ satisfying the conclusion of Theorem 8 is $x_{0}=0$.

\section{Weak equilibrium problems}

Theorem 10. Let $X, Z$ and $V$ be topological spaces and $(Y, D ; \Gamma)$ be a $G$ convex space. Let $C: Z \multimap V, F: Y \times Z \multimap V, P: X \multimap Z$, be three mappings and $\rho$ be a relation on $2^{V}$. Assume that:

(i) $P$ is u.s.c.;

(ii) for each $z \in Z$ the set $\left\{y \in Y: F(y, z) \rho^{c} C(z)\right\}$ is $G$-convex;

(iii) for each $u \in D$ the set $\{z \in Z: F(u, z) \rho C(z)\}$ is closed;

(iv) there exists a compact mapping $T \in \mathfrak{B}(Y, X)$ such that for each $y \in Y$ and any $x \in T(y)$ there exists $z \in P(x)$ such that $F(y, z) \rho C(z)$.

Then there exists $x_{0} \in X$ such that for each $u \in D$ there is $z \in P\left(x_{0}\right)$ satisfying $F(u, z) \rho C(z)$.

Proof. Define the mapping $Q: Y \multimap Z$ by

$$
Q(y)=\left\{z \in Z: F(y, z) \rho^{c} C(z)\right\} .
$$

Suppose that the conclusion does not hold. Then for each $x \in X$ there exists $u \in D$ such that $F(u, z) \rho^{c} C(z)$ for all $z \in P(x)$, that is, $P(x) \subseteq Q(u)$. By (ii), $Q$ has $G$-convex values and by (iii), $Q_{\mid D}$ has open values. The requirements of Corollary 5 are verified, hence there exists $(\widetilde{x}, \widetilde{y}) \in X \times Y$ such that $\widetilde{x} \in T(\widetilde{y})$ 
and $P(\widetilde{x}) \subseteq Q(\widetilde{y})$. Then for each $z \in P(\widetilde{x})$ we have $F(\widetilde{y}, z) \rho^{c} C(z)$, but this contradicts (iv).

Theorem 11. Let $X, Y, Z, V, C, F$ and $P$ be as in Theorem 10. Suppose that $D$ is dense in $Y, P$ has compact values, the mapping $S: Y \multimap Z$ defined by $S(y)=\{z \in Z: F(y, z) \rho C(z)\}$ is closed and conditions (i), (ii) and (iv) in Theorem 10 are fulfilled. Then there exists $x_{0} \in X$ such that for each $y \in Y$ there is $z \in P\left(x_{0}\right)$ satisfying $F(y, z) \rho C(z)$, i.e., $x_{0}$ is a solution of the weak $\rho$-equilibrium problem with respect to $P,\left(V I_{2}\right)$.

Proof. Since $S$ is a closed mapping, it has closed values hence condition (iii) in Theorem 10 holds. By the previous theorem there is an $x_{0} \in X$ which is solution of the weakly $\rho$-equilibrium problem with respect to $P,\left(V I_{1}\right)$. Let $y \in Y$. Since $D$ is dense in $Y$ there is a net $\left\{u_{\alpha}\right\}_{\alpha \in \Lambda}$ converging to $y$. For each $\alpha \in \Lambda$ there is $z_{\alpha} \in P\left(x_{0}\right)$ such that $F\left(u_{\alpha}, z_{\alpha}\right) \rho C\left(z_{\alpha}\right)$. Since $P\left(x_{0}\right)$ is compact we may suppose that $\left\{z_{\alpha}\right\}_{\alpha \in \Lambda}$ converges to a point $z \in P\left(x_{0}\right)$. Therefore $\left\{\left(u_{\alpha}, z_{\alpha}\right)\right\}_{\alpha \in \Lambda}$ is a net in $\operatorname{Gr} S$ converging to $(y, z)$. Since $S$ is closed, it follows that $z \in S(y)$, that is $F(y, z) \rho C(z)$. Hence $x_{0}$ is a solution of the weak $\rho$-equilibrium problem with respect to $P,\left(V I_{2}\right)$.

In the next results from this section we will assume that $\left(Z, D^{\prime} ; \Gamma^{\prime}\right)$ is a $G$-convex spaces and $X, Y, V, C, F$ and $P$ are as in Theorem 10 .

Theorem 12. Suppose that:

(i) $P$ is u.s.c.;

(ii) $C$ is a closed mapping;

(iii) for each $u \in D, F(u, \cdot)$ is l.s.c.;

(iv) one of the following situations holds:

(iv 1 ) for each $z \in Z, F(\cdot, z)$ is G-strongly quasiconvex;

$\left(\mathrm{iv}_{2}\right) V$ is a $G$-convex space and for each $z \in Z, V \backslash C(z)$ is $G$-convex and $F(\cdot, z) G$-quasiconvex;

$\left(\right.$ iv $\left._{3}\right) V$ is a topological vector space and for each $z \in Z, C(z)$ is a convex cone and $F(\cdot, z)$ is $C(z)-G$-quasiconvex;

(v) there exists a compact mapping $T \in \mathfrak{B}(Y, X)$ such that for each $y \in Y$ and any $x \in T(y)$ there exists $z \in P(x)$ such that $F(y, z) \subseteq C(z)$.

Then there exists $x_{0} \in X$ such that for each $u \in D$ there is $z \in P\left(x_{0}\right)$ satisfying $F(u, z) \subseteq C(z)$.

Proof. The mappings $P, F$ and $C$ satisfy all the requirements of Theorem 10 when $\rho=\rho_{1}$. In order to prove that for each $u \in D$ the set $\{z \in Z: F(u, z) \subseteq$ $C(z)\}$ is closed see the first part of the proof of Theorem 8. In order to prove that for each $z \in Z$ the set $\{y \in Y: F(y, z) \nsubseteq C(z)\}$ is $G$-convex see the last part of the same theorem. The conclusion follows from Theorem 10.

Theorem 13. Suppose that $D$ is dense in $Y$, conditions (i), (ii), (iv) and (v) in Theorem 12 are fulfilled and condition (iii) is replaced by the following condition: 
(iii') $F$ is l.s.c. on $Y \times Z$.

Then there exists $x_{0} \in X$ such that for each $y \in Y$ there is $z \in P\left(x_{0}\right)$ satisfying $F(y, z) \subseteq C(z)$.

Proof. It's clear that condition (iii') implies condition (iii) in Theorem 12. According to Theorem 11 it suffices to prove that the map $S: X \multimap Y$ defined by $S(y)=\{z \in Z: F(y, z) \subseteq C(z)\}$ is closed. Let $(y, z) \in \overline{G r S}$. Then there exists a net $\left\{\left(y_{\alpha}, z_{\alpha}\right)\right\}_{\alpha \in \Lambda}$ in $\operatorname{Gr} S$ such that $\left(y_{\alpha}, z_{\alpha}\right) \rightarrow(y, z)$. Let $v \in F(y, z)$ be arbitrarily fixed. Since $F$ is l.s.c. on $Y \times Z$, by Lemma 1 (iv), there is a net $\left\{v_{\alpha}\right\}_{\alpha \in \Lambda}$ such that $v_{\alpha} \in F\left(y_{\alpha}, z_{\alpha}\right)$ and $v_{\alpha} \rightarrow v$. For each $\alpha \in \Lambda$, since $\left(y_{\alpha}, z_{\alpha}\right) \in G r S$, we have $v_{\alpha} \in F\left(y_{\alpha}, z_{\alpha}\right) \subseteq C\left(z_{\alpha}\right)$. Since $C$ is a closed mapping we infer that $v \in C(z)$, hence $(y, z) \in G r S$. Therefore $S$ is closed.

Theorem 14. Suppose that

(i) $P$ is u.s.c.;

(ii) $C$ is a closed mapping;

(iii) for each $u \in D, F(u, \cdot)$ is u.s.c.;

(iv) one of the following situations holds:

(iv 1 ) for each $z \in Z, F(\cdot, z)$ has $G$-convex cofibers;

$\left(\mathrm{iv}_{2}\right) V$ is a topological vector space and for each $z \in Z, C(z)$ is a convex cone and $F(\cdot, z)$ is $C(z)-G$-quasiconvex-like;

(v) there exists a compact mapping $T \in \mathfrak{B}(Y, X)$ such that for each $y \in Y$ and any $x \in T(y)$ there exists $z \in P(x)$ such that $F(y, z) \cap C(z) \neq \emptyset$.

Then there exists $x_{0} \in X$ such that for each $u \in D$ there is $z \in P\left(x_{0}\right)$ satisfying $F(u, z) \cap C(z) \neq \emptyset$.

Proof. Arguing as in the proof of Theorem 12 one can show that the mappings $P, F, C$ satisfy all the requirements of Theorem 10 when $\rho=\rho_{2}$. Conclusion follows from Theorem 10.

Theorem 15. Suppose that $D$ is dense in $Y, V$ is compact, conditions (i), (ii), (iv) and (iv) in Theorem 14 are fulfilled and condition (iii) is replaced by the following condition:

(iii') $F$ is a closed mapping.

Then there exists $x_{0} \in X$ such that for each $y \in Y$ there is $z \in P\left(x_{0}\right)$ satisfying $F(y, z) \cap C(z) \neq \emptyset$.

Proof. According to Theorem 14 it suffices to prove that the map $S: Y \multimap Z$ defined by $S(y)=\{z \in Z: F(y, z) \cap C(z) \neq \emptyset\}$ is closed. Let $(y, z) \in \overline{G r S}$. Then there exists a net $\left\{\left(y_{\alpha}, z_{\alpha}\right)\right\}_{\alpha \in \Lambda}$ in $G r S$ such that $\left(y_{\alpha}, z_{\alpha}\right) \rightarrow(y, z)$. For each $\alpha \in \Lambda$ let $v_{\alpha} \in F\left(y_{\alpha}, z_{\alpha}\right) \cap C\left(z_{\alpha}\right)$. Since $V$ is compact we may suppose that $\left\{v_{\alpha}\right\}$ converges to a $v \in V$. Since $F$ and $C$ are closed it follows that $v \in F(y, z) \cap C(z)$, hence $(y, z) \in G r S$.

The following result is a version of Theorem 4 in [5]. It can be obtained from Theorem 10 taking $Y=Z$ and $T(y)=\{y\}$ for all $y \in Y$. In the same manner 
from Theorems 11, 12, 13, 14 and 15 we can obtain versions of Theorems 5, 6, 7, 9 and 10 in the mentioned paper.

Theorem 16. Let $(Y, D ; \Gamma),\left(Z, D^{\prime} ; \Gamma^{\prime}\right)$ be $G$-convex spaces, $Y$ being compact, $V$ be a topological space, $\rho$ be a relation on $2^{V}$ and $P: Y \multimap Z, F: Y \times Z \multimap V$, $C: Y \multimap Z$ be three mappings satisfying the following conditions:

(i) $P$ is u.s.c.;

(ii) for each $z \in Z$ the set $\left\{y \in Y: F(y, z) \rho^{c} C(z)\right\}$ is $G$-convex;

(iii) for each $u \in D$ the set $\{z \in Z: F(u, z) \rho C(z)\}$ is closed;

(iv) for each $y \in Y$ there exists $z \in P(y)$ such that $F(y, z) \rho C(y)$.

Then there exists $y_{0} \in Y$ such that for each $u \in D$ there is $z \in P\left(y_{0}\right)$ satisfying $F(u, z) \rho C(z)$.

\section{References}

[1] Q. H. Ansari, W. Oettle, and D. Schläger, A generalization of vectorial equilibria, Math. Methods Oper. Res. 46 (1997), no. 2, 147-152.

[2] Q. H. Ansari, S. Schaible, and J. C. Yao, System of vector equilibrium problems and its applications, J. Optim. Theory Appl. 107 (2000), no. 3, 547-557.

[3] M. Balaj, An intersection theorem with applications in minimax theory and equilibrium problem, J. Math. Anal. Appl. 336 (2007), no. 1, 363-371.

[4] Coincidence and maximal element theorems and their applications to generalized equilibrium problems and minimax inequalities, Nonlinear Anal. 68 (2008), no. 12, 3962-3971.

[5] M. Balaj and D. O'Regan, Weak-equilibrium problems in G-convex spaces, Rend. Circ. Mat. Palermo (2) 57 (2008), no. 1, 103-117.

[6] H. Ben-El-Mechaiekh, S. Chebbi, M. Florenzano, and J.-V. Llinares, Abstract convexity and fixed points, J. Math. Anal. Appl. 222 (1998), no. 1, 138-150.

[7] T.-H. Chang and C.-L. Yen, KKM property and fixed point theorems, J. Math. Anal. Appl. 203 (1996), no. 1, 224-235.

[8] X. P. Ding, Genralized KKM type theorems in FC-spaces with applications. I, J. Global Optim. 36 (2006), no. 4, 581-596.

[9] __ Generalized KKM type theorems in FC-spaces with applications. II, J. Global Optim. 38 (2007), no. 3, 367-385.

[10] X. P. Ding and T. M. Ding, KKM type theorems and generalized vector equilibrium problems in noncompact FC-spaces, J. Math. Anal. Appl. 331 (2007), no. 2, 1230-1245.

[11] X. P. Ding and Y. J. Park, Fixed points and generalized vector equilibrium problems in generalized convex spaces, Indian J. Pure Appl. Math. 34 (2003), no. 6, 973-990.

[12] _ Generalized vector equilibrium problems in generalized convex spaces, J. Optim. Theory Appl. 120 (2004), no. 2, 327-353.

[13] M. Fakhar and J. Zafarani, Generalized vector equilibrium problems for pseudomonotone multivalued bifunctions, J. Optim. Theory Appl. 126 (2005), no. 1, 109-124.

[14] M. Fang and N. Huang, KKM type theorems with applications to generalized vector equilibrium problems in FC-spaces, Nonlinear Anal. 67 (2007), no. 3, 809-817.

[15] J. Y. Fu, Generalized vector quasi-equilibrium problems, Math. Methods Oper. Res. 52 (2000), no. 1, 57-64

[16] M. Lassonde, Fixed points for Kakutani factorizable multifunctions, J. Math. Anal. Appl. 152 (1990), no. 1, 46-60.

[17] L. J. Lin, Q. H. Ansari, and J. Y. Wu, Geometric properties and coincidence theorems with applications to generalized vector equilibrium problems, J. Optim. Theory Appl. 117 (2003), no. 1, 121-137. 
[18] L. J. Lin and H. L. Chen, The study of KKM theorems with applications to vector equilibrium problems with implicit vector variational inequalities problems, J. Global Optim. 32 (2005), no. 1, 135-157.

[19] L. J. Lin and W. S. Du, Systems of equilibrium problems with applications to generalized Ekeland's variational principle and systems of semi-infinite problems, J. Global Optim. (2007).

[20] L. J. Lin and W. P. Wan, KKM type theorems and coincidence theorems with applications to the existence of equilibria, J. Optim. Theory Appl. 123 (2004), no. 1, 105-122.

[21] L. J. Lin, Z. T. Yu, and G. Kassay, Existence of equilibria for multivalued mappings and its application to vectorial equilibria, J. Optim. Theory Appl. 114 (2002), no. 1, 189-208.

[22] Z. D. Mitrovic, On scalar equilibrium problem in generalized convex spaces, J. Math. Anal. Appl. 330 (2007), no. 1, 451-461.

[23] S. Park, Fixed points of the better admissible multimaps, Msath. Sci. Res. Hot-Line 1 (1997), no. 9, 1-6.

[24] Foundations of the KKM theory via coincidences of composites of upper semicontinuous maps, J. Korean Math. Soc. 31 (1994), no. 3, 493-519.

[25] _ Fixed points of better admissible maps on generalized convex spaces, J. Korean Math. Soc. 37 (2000), no. 6, 885-899.

[26] _ New subclasses of generalized convex spaces, Fixed point theory and applications (Chinju, 1998), 91-98, Nova Sci. Publ., Huntington, NY, 2000.

[27] _ Remarks on fixed point theorems for generalized convex spaces, Fixed point theory and applications (Chinju, 1998), 135-144, Nova Sci. Publ., Huntington, NY, 2000.

[28] _ Fixed point theorems in locally G-convex spaces, Nonlinear Anal. 48 (2002), no. 6, Ser. A: Theory Methods, 869-879.

[29] , Remarks on equilibria for g-monotone maps on generalized convex spaces, J. Math. Anal. Appl. 269 (2002), no. 1, 244-255.

[30] S. Park and H. Kim, Admissible classes of multifunctions on generalized convex spaces, Proc. Coll. Natur. Sci. Seoul National University 18 (1993), 1-21.

[31] N. X. Tan and P. N. Tinh, On the existence of equilibrium points of vector functions, Numer. Funct. Anal. Optim. 19 (1998), no. 1-2, 141-156.

\author{
Mircea Balaj \\ Department of Mathematics \\ UNIVERSITY OF ORADEA \\ OradeA, 411087, OradeA, Romania \\ E-mail address: mbalaj@uoradea.ro \\ DONAL O'REGAN \\ Department of Mathematics \\ NATIONAL UNIVERSiTy OF IRELAND \\ GALWAY, IRELAND \\ E-mail address: donal.oregan@nuigalway.ie
}

